\title{
Optimal Signal to Noise Ratio in Feedback over Communication Channels with Memory
}

\author{
A.J. Rojas, J.S. Freudenberg, J.H. Braslavsky and R.H. Middleton
}

\begin{abstract}
Communication channels impose a number of obstacles to feedback control, such as delay, noise, and constraints in communication data-rate. One alternate line of recent work considers the problem of feedback stabilization subject to a constraint in the signal-to-noise ratio (SNR). It has been shown for continuous-time systems that the optimal control problem arising in achieving minimal SNR can be formulated as a linear quadratic Gaussian (LQG) control problem with weights chosen as in the loop transfer recovery (LTR) technique. The present paper extends such LQG/LTR formulation to discretetime systems with feedback over channels with memory. By using such formulation, we derive exact expressions for the LTI controller and loop sensitivity functions that achieve minimal SNR under the effect of time-delay, non minimum phase zeros and colored additive noise. For the minimum-phase case with white noise and no time delay, we show that the optimal feedback loop obtained after applying LTR has a structure equivalent to that of a communication channel with feedback from the output to the input.
\end{abstract}

\section{INTRODUCTION}

The study of control problems with feedback over communication channels has grown increasingly in recent years; see for example [1] and references therein. Communication channels impose additional limitations to feedback, such as constraints in data-rate and bandwidth, and effects of noise and time delays. One line of recent works introduced a framework to study stabilisability with feedback over channels with a signal to noise ratio (SNR) constraint [10], [5], [12]. These papers determined the minimal SNR required to stabilize an unstable plant over an additive white Gaussian noise (AWGN) channel. For the case of linear time invariant (LTI) controllers, these conditions match precisely those derived in [13] by application of Shannon's theorem on the capacity of a communication channel [7, $§ 10.3]$. A distinctive characteristic of the SNR approach is that it is a linear formulation, suited for the analysis of robustness using welldeveloped tools [16].

The presence of time delay increases the SNR required to stabilize (using LTI control) an unstable system, as shown in [5], [12]. These papers treated time delays in discretetime, wherein they appear as an increase in the relative degree of a finite dimensional plant. Bounds for the required SNR with time delays in continuous-time are given in [11],

J.S Freudenberg is with the Department of Electrical Engineering and Computer Science, The University of Michigan, Ann Arbor, MI, 481092122, U.S.A. Email: jfr@eecs . umich. edu.

A.J. Rojas, J.H. Braslavsky and R.H. Middleton are with the Centre for Complex Dynamic Systems and Control, The University of Newcastle, 2308, Australia. Emails: alejandro.rojas@studentmail. newcastle.edu.au, julio.braslavsky@newcastle.edu.au, richard.middletonenewcastle.edu.au with similar conclusions, albeit with considerably greater technical complexity due to the infinite dimensionality of the relevant dynamic operators. In the subsequent paper [3], the authors derive exact closed-form expressions for the SNR required for closed-loop stability with continuous-time timedelays. The paper [3] studies output feedback over additive coloured Gaussian noise channels with memory, in the sense that the channel has a transfer function used to model a bandwidth limitation in the continuous-time case.

Different techniques are used in the papers [10], [5], [12] depending on whether stabilization is achieved by state feedback or by output feedback. A common framework for both state and output feedback cases is proposed in [4], where it is shown that both problems can be solved as a linear quadratic Gaussian (LQG) optimization. To be specific, the $\mathscr{H}_{2}$ optimal control problem arising in minimal SNR control may be posed as an LQG problem with weights chosen as in the loop transfer recovery (LTR) technique (see [9], [14], [8], [15], [6]). Doing so not only allows a unified treatment of the state and output feedback cases, but also suggests, as pointed out in [4], how performance considerations may be analyzed in addition to stabilization. Also, posing the problem as one of LQG provides insight into the structure of the optimal controller, a fact that we shall highlight in the present paper.

Our goal in the present paper is to reformulate the problems solved in [3] as LQG problems as motivated by the results of [4]. The present results differ from those of [4] in that we consider channels with memory, and treat discretetime systems. Focusing on discrete-time allows us to consider the effects of delay in a simple way, as noted above, and also (as we shall see) to pose a problem that admits exact, as opposed to asymptotic, expressions for the controller that optimises SNR. By using these expressions, we show that, in the non-minimum phase case with white noise and no delays, the optimal LTR solution can be interpreted as a communication channel with feedback from the output to the input. The specific structure of this feedback is that of a linear quadratic optimal observer.

The rest of the paper is organized as follows: Section II introduces some preliminary concepts and definitions. Section III reviews discrete-time observers and the LQG/LTR technique. Section IV analyzes the closed loop stabilisability problem when an ACGN channel with memory is located between the plant and the controller. Section V presents an interpretation of the resulting optimal feedback loop for an AWGN channel in terms of an equivalent optimal estimator. Section VI gives the paper conclusions. 


\section{PRELIMINARIES}

We consider the discrete-time feedback system depicted in Figure 1, where $G(z)$ and $C(z)$ are scalar proper transfer functions representing the plant and controller. The feedback loop is closed over a noisy channel with memory, characterized by the scalar proper transfer functions $F(z)$ and $H(z)$. The noise $n_{k}$ is assumed white and Gaussian with variance $\sigma^{2}$. We assume that $C(z)$ is such that the closed-loop system is stable in the sense that, for any distribution of initial conditions, the distribution of all signals in the loop will converge exponentially rapidly to a stationary distribution.

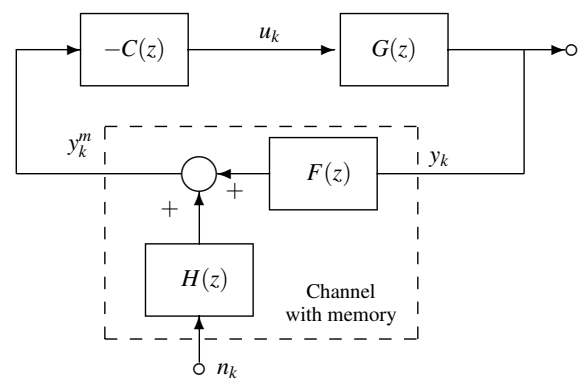

Fig. 1. Control system with feedback over a noisy channel with memory.

The channel input power, defined by $\mathscr{E}\left\{y_{k}^{2}\right\}$, where $\mathscr{E}$ denotes expectation, is required to satisfy the constraint

$$
\mathscr{P}_{d}>\mathscr{E}\left\{y_{k}^{2}\right\}
$$

for some predetermined power level $\mathscr{P}_{d}$. Under reasonable stationarity assumptions [2, $\$ 4.4]$, the power in the channel input may be computed from its spectral density as

$$
\mathscr{E}\left\{y_{k}^{2}\right\}=\frac{1}{2 \pi} \int_{-\pi}^{\pi} S_{y}(\omega) d \omega=\frac{1}{2 \pi} \int_{-\pi}^{\pi}\left|T_{y n}\right|^{2} \sigma^{2} d \omega,
$$

where

$$
T_{y n}(z)=-\frac{C(z) G(z)}{1+C(z) G(z) F(z)} H(z)
$$

is the transfer function that relates $y_{k}$ with $n_{k}$. Since the feedback system is stable, then

$$
\mathscr{E}\left\{y_{k}^{2}\right\}=\left\|T_{y n}\right\|_{\mathscr{H}_{2}}^{2} \sigma^{2}
$$

Thus, the power constraint (1) at the input of the channel translates to the SNR bound on the $\mathscr{H}_{2}$ norm of $T_{y n}$

$$
\frac{\mathscr{P}_{d}}{\sigma^{2}}>\left\|T_{y n}\right\|_{\mathscr{H}_{2}}^{2} \text {. }
$$

As a consequence, the problem of stabilization subject to the constraint (1) leads to an $\mathscr{H}_{2}$ optimization. Such optimization can be approached as an LQG control problem with weights chosen as in the LTR technique [4]. In the case considered here, in which the channel is located between measurements and controller (see Figure 1), the LTR technique consists of first designing an optimal state estimator based on the given noise statistical properties, and then constructing an observer-based compensator with a state feedback law $u_{k}=K \hat{x}_{k}$ using the state estimate $\hat{x}_{k}$. The gain $K$ is chosen as the linear quadratic optimal cheap control gain. We next review optimal observers and the above LTR technique for discrete-time systems.

\section{State Estimation AND Loop TRANSFER RECOVERY}

There are two distinctive characteristics of the LTR technique when dealing with discrete-time systems. First, a state observer can be implemented in a filtering form or in a predicting form. Second, the computation of the optimal cheap control state feedback gain for LTR can be done exactly, as opposed to asymptotically, as is the case for continuoustime systems in general [9]. In this section we first review these two types of discrete-time optimal observers, and then discuss the LTR technique.

Consider the general discrete-time system

$$
\begin{aligned}
x_{k+1} & =A x_{k}+B u_{k}+w_{k}, \\
y_{k}^{m} & =C x_{k}+v_{k},
\end{aligned}
$$

where $(A, B, C)$ is controllable and observable, $x_{k} \in \mathbb{R}^{n}$ is the state vector, $u_{k} \in \mathbb{R}$ is the control input and $y_{k}^{m} \in \mathbb{R}$ is the output. The process and measurement noises $w_{k} \in \mathbb{R}^{n}$ and $v_{k} \in \mathbb{R}$ are assumed zero mean Gaussian processes with covariance matrix

$\mathscr{E}\left\{\left[\begin{array}{l}w_{k} \\ v_{k}\end{array}\right]\left[\begin{array}{ll}w_{l}^{T} & v_{l}\end{array}\right]\right\}=\left[\begin{array}{ll}W & S \\ S^{T} & V\end{array}\right] \delta_{k l}, \quad \delta_{k l}= \begin{cases}1 & \text { if } k=l, \\ 0 & \text { if } k \neq l .\end{cases}$

A general discrete-time observer for this system is defined by the equation

$$
\hat{x}_{k+1 \mid k}=A \hat{x}_{k \mid k-1}+B u_{k}+K_{p}\left(y_{k}^{m}-C \hat{x}_{k \mid k-1}\right),
$$

where the gain $K_{p}$ is chosen such that the matrix $A-K_{p} C$ has stable eigenvalues.

\section{A. Filtering Observer-Based Compensator}

A filtering observer is defined by (4) with $K_{p}=A K_{f}$, and the state estimate given by

$$
\hat{x}_{k \mid k}=\hat{x}_{k \mid k-1}+K_{f}\left(y_{k}^{m}-C \hat{x}_{k \mid k-1}\right) \text {. }
$$

The state estimate is updated by the current measurement $y_{k}^{m}$. In the optimal filtering observer, the gain $K_{f}$ is given by

$$
K_{f}=\left(A \Sigma C^{T}+S\right)\left(C \Sigma C^{T}+W\right)^{-1},
$$

where $\Sigma$ is the positive semidefinite solution to the discretetime Riccati equation

$$
\begin{aligned}
\Sigma=A \Sigma A^{T}-\left(A \Sigma C^{T}+S\right)\left(C \Sigma C^{T}\right. & +W)^{-1} \\
& \times\left(C \Sigma A^{T}+S\right)+V .
\end{aligned}
$$

When this observer is considered together with the linear control law

$$
u_{k}=-K \hat{x}_{k \mid k}
$$

the transfer function of the resulting filtering observer-based compensator is given by

$$
C_{f}(z)=z K\left(z I-\left(I-K_{f} C\right)(A-B K)\right)^{-1} K_{f} .
$$




\section{B. Predicting Observer-Based Compensator}

A predicting observer is defined by (4) with $K_{p}=A K_{f}$, and the state estimate directly given by $\hat{x}_{k \mid k-1}$. In the optimal predicting observer, $K_{f}$ is given by (6), (7), as for the optimal filtering observer.

When the predicting observer is combined with the linear control law

$$
u_{k}=-K \hat{x}_{k \mid k-1},
$$

we obtain a predicting observer-based compensator with transfer function

$$
C_{p}(z)=K\left(z I-A+B K+K_{p} C\right)^{-1} K_{p} .
$$

\section{Loop Transfer Recovery}

The process of recovering, in the framework of the LQG/LTR technique we are considering, will result in the state feedback gain $K$. Define the quadratic cost index to be minimized as

$$
J=\sum_{k=0}^{\infty} x_{k}^{T} Q x_{k}+R u_{k}^{2},
$$

where $R>0$ and $Q \geq 0$. It is known that the optimal state feedback gain is given by

$$
K=\left(R+B^{T} P B\right)^{-1} B^{T} P A,
$$

where $P$ is the solution to the discrete-time Riccati equation

$$
P=A^{T} P A-A^{T} P B\left(R+B^{T} P B\right)^{-1} B^{T} P A+Q .
$$

Recovery of the observer loop design is achieved whenever $R=0$ (cheap control) and $Q=C^{T} C$.

\section{OPTIMAL SNR VIA LQG/LTR}

We return to the system depicted in Figure 1. We assume the LTI filters $F(z)$ and $H(z)$ are both biproper and stable; $H(z)$ is minimum phase, but $F(z)$ is permitted to be nonminimum phase. We assume that the plant $G(z)$ is unstable and possibly non-minimum phase. The state space description of the augmented system including $G, F, H$ as in Figure 1 is

$$
\begin{gathered}
x_{k+1}=\left[\begin{array}{c}
x_{k+1}^{G} \\
x_{k+1}^{F} \\
x_{k+1}^{H}
\end{array}\right]=\underbrace{\left[\begin{array}{ccc}
A_{G} & 0 & 0 \\
B_{F} C_{G} & A_{F} & 0 \\
0 & 0 & A_{H}
\end{array}\right]}_{A} x_{k}+ \\
+\underbrace{\left[\begin{array}{c}
B_{G} \\
0 \\
0
\end{array}\right]}_{C} u_{B}^{\left[\begin{array}{c}
0 \\
0 \\
B_{H}
\end{array}\right]} n_{k} \\
y_{k}^{m}=\underbrace{\left[\begin{array}{ll}
D_{F} C_{G} & C_{F} \\
C_{H}
\end{array}\right]}_{B_{n}} x_{k}+\underbrace{D_{H}}_{D} n_{k},
\end{gathered}
$$

where $G \triangleq\left(A_{G}, B_{G}, C_{G}\right), F \triangleq\left(A_{F}, B_{F}, C_{F}, D_{F}\right)$ and $H \triangleq$ $\left(A_{H}, B_{H}, C_{H}, D_{H}\right)$. We consider the augmented system (14) as a specific case of the general system (3).

Consider the noise $n_{k}$ to be white and Gaussian and, without loss of generality, take $\mathscr{E}\left\{n_{k} n_{l}^{T}\right\}=\delta_{k l}$. In regards to (3), we have that $v_{k}=D n_{k}$ and $w_{k}=B_{n} n_{k}$ (the measurement and process noises), and the covariance matrix is

$$
\mathscr{E}\left\{\left[\begin{array}{c}
w_{k} \\
v_{k}
\end{array}\right]\left[\begin{array}{ll}
w_{l}^{T} & v_{l}^{T}
\end{array}\right]\right\}=\left[\begin{array}{cc}
B_{n} B_{n}^{T} & B_{n} D^{T} \\
D B_{n}^{T} & D D^{T}
\end{array}\right] \delta_{k l} .
$$

To state our results, we now need to define terms that appear when the plant $G$ or the filter $F$ contains nonminimum phase zeros.

\section{A. Plant Non-Minimum Phase (NMP) Zeros}

From [15], in order to deal with NMP zeros of $G$, notice that $G$ can be rewritten as

$$
G=C_{G}\left(z I-A_{G}\right)^{-1} B_{G}=C_{a}(z) C_{m}\left(z I-A_{G}\right)^{-1} B_{G},
$$

where $C_{a}(z)=C_{a 1}(z) C_{a 2}(z) \cdots C_{a r}$ and

$$
C_{m}=C_{m}^{r}
$$

are defined recursively by

$$
\begin{aligned}
C_{a i} & =I-\left(\frac{a_{i} \bar{a}_{i}-1}{a_{i}+1}\right)\left(\frac{z+1}{z \bar{a}_{i}-1}\right) \bar{\eta}_{i} \eta_{i}^{T} \\
C_{m}^{i} & =C_{m}^{i-1}-\left(\frac{a_{i} \bar{a}_{i}-1}{\bar{a}_{i}+1}\right) \bar{\eta}_{i} \varepsilon_{i}^{T}\left(A_{G}+I\right), \forall i=1, \cdots, r,
\end{aligned}
$$

where $\bar{\eta}$ is the complex conjugate of $\eta$, and $a_{i}$ are the NMP zeros of the plant, $r$ is the number of NMP zeros, and the vectors $\varepsilon_{i}, \eta_{i}$ are the solutions to

$$
\left[\begin{array}{ll}
\varepsilon_{i}^{T} & \eta_{i}^{T}
\end{array}\right]\left[\begin{array}{cc}
a_{i} I-A_{G} & -B_{G} \\
-C_{m}^{i-1} & 0
\end{array}\right]=0 .
$$

\section{B. Channel NMP zeros}

If the filter $F$ contains $q$ NMP zeros, labeled as $f_{i}, \forall i=$ $1, \cdots, q$, we can write $F$ as

$$
F(z)=L_{F} \tilde{F}(z)
$$

where

$$
L_{F}=\prod_{i=1}^{q}=\frac{z-f_{i}}{1-z \bar{f}_{i}}
$$

is the all pass term containing all $q$ NMP zeros. If the term $L_{F}$ is now included into the plant model, as say $\tilde{G}=L_{F} G$, then the overall problem of SNR minimization can now be analyzed for the triplet $(\tilde{G}, \tilde{F}, H)$ instead of the original $(G, F, H)$. Since $L_{F}$ is all pass it will not modify the power constraint at the channel input and the NMP zeros can now be dealt with as if they are plant NMP zeros. 


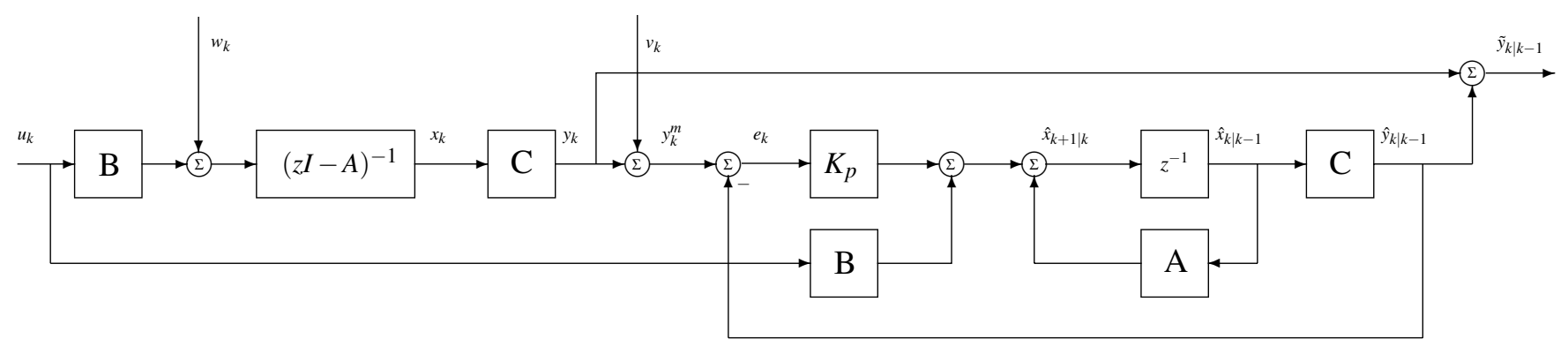

Fig. 2. Predicting observer.

\section{Main Result}

For the LTR technique considered, we obtain the optimal filtering and predicting observers (8), (10) directly from the definition of the augmented system (14), (15). We specify the observer gain

$$
K_{p}=A K_{f}=\left[\begin{array}{c}
K_{p}^{G} \\
K_{p}^{F} \\
K_{p}^{H}
\end{array}\right]
$$

with $K_{p}^{G}, K_{p}^{F}, K_{p}^{H}$ with dimensions compatible with those of the augmented state vector in (14), and $K_{f}$ given by (6), (7) for the augmented system (14), (15).

For the computation of the optimal cheap state feedback gain $K$ in (12), (13), we use the cost index

$$
J=\sum_{k=0}^{\infty} y_{k}^{2}=\sum_{k=0}^{\infty} x_{k}^{T}\left[\begin{array}{c}
C_{G}^{T} \\
0 \\
0
\end{array}\right]\left[\begin{array}{lll}
C_{G} & 0 & 0
\end{array}\right] x_{k} .
$$

which corresponds to the cost index in (11). An exact (not asymptotic) expression for the optimal cheap gain $K$, with

$$
Q=\left[\begin{array}{c}
C_{G}^{T} \\
0 \\
0
\end{array}\right]\left[\begin{array}{lll}
C_{G} & 0 & 0
\end{array}\right]
$$

and $R=0$, is obtained by direct application of a result from [15].

Lemma 1: Consider the system (14), and assume that the relative degree of $G$ equals $l$; that is, $C B=C A B=\cdots=$ $C A^{l-2} B=0, C A^{l-1} B \neq 0$. Then, the optimal cheap state feedback gain (12), (13), with $Q$ given by (24) and $R=0$, is

$$
K=\left(C_{m} A_{G}^{l-1} B_{G}\right)^{-1}\left[C_{m} A_{G}^{l} \quad 0 \quad 0\right],
$$

where $C_{m}$ is given by (17), (18).

Proof: See [15, Theorem 2.1].

Define the observer loop sensitivity function as

$$
S_{o b}(z)=(1+\mathscr{H}(z))^{-1}, \quad \mathscr{H}(z)=C(z I-A)^{-1} K_{p},
$$

and define similarly

$$
S_{o}(z)=\left(1+L_{o}(z)\right)^{-1}, \quad L_{o}(z)=F(z) G(z) C(z),
$$

where $C(z)$ is the compensator in Figure 1.

Theorem 2: Assume that $H$ contains no NMP zeros. If the observer design is performed using covariance matrix defined in (15), the resulting gain $K_{p}$ is defined as in (22), and recovery is applied using $\left[\begin{array}{lll}C_{m} & 0 & 0\end{array}\right]$, then the optimal output open loop function and optimal output feedback loop sensitivity function are

$$
L_{o}=\left[\mathscr{H}-E_{d}\right]\left[1+E_{d}\right]^{-1}, \quad S_{o}=\left[1+E_{d}\right] S_{o b},
$$

where

a) For the filtering observer-based compensator (8),

$$
\begin{aligned}
E_{d}=C_{F} \Phi_{F} & K_{p}^{F}+C_{H} \Phi_{H} K_{p}^{H} \\
& +F\left(C_{G}-\frac{1}{z^{l-1}} C_{a} C_{m} A_{G}^{l-1}\right) \Phi_{G} K_{p}^{G} .
\end{aligned}
$$

b) For the predicting observer-based compensator (10),

$$
\begin{aligned}
E_{d}=C_{F} \Phi_{F} K_{p}^{F} & +C_{H} \Phi_{H} K_{p}^{H} \\
& +F\left(C_{G}-\frac{1}{z^{l}} C_{a} C_{m} A_{G}^{l}\right) \Phi_{G} K_{p}^{G} .
\end{aligned}
$$

In both cases $\Phi_{G}=\left(z I-A_{G}\right)^{-1}, \Phi_{F}=\left(z I-A_{F}\right)^{-1}$ and $\Phi_{H}=\left(z I-A_{H}\right)^{-1}$.

Proof: See Appendix.

From [3] and Theorem 2 we can obtain an expression for the optimal SNR required for stability.

Corollary 3: The feedback loop in Figure 1 can be stabilized if and only if the SNR (2) satisfies

$$
\frac{\mathscr{P}_{d}}{\sigma^{2}}>\left\|\left[1+E_{d}(z)\right] S_{o b}(z) G(z) \hat{C}(z) H(z)\right\|_{\mathscr{H}_{2}}^{2} .
$$

where $\hat{C}(z)$ is the optimal controller that achieves the minimum SNR (either predicting or filtering).

The following example includes consideration of a plant with repeated poles, a case hard to analyze from the algebraic perspective presented in [3].

Example 1: Consider the following plant, filter $F$ and filter $H$ :

$G(z)=\frac{z+0.1}{z^{2}+2 \alpha z+4}, \quad F(z)=\frac{5}{8} \frac{z-0.2}{z-0.5}, \quad H(z)=\frac{7}{9} \frac{z-0.1}{z-0.3}$,

with $\alpha$ in $[-2,2]$. Notice that under this definition the plant will show a pair of complex conjugate poles with real part $\alpha$ and imaginary part $\sqrt{4-\alpha^{2}}$ and whenever $|\alpha|=2$ the plant will have repeated poles.

For this example it is assumed that the associated computational time is negligible. Therefore the filtering compensator is the working choice in order to compute the SNR required for stabilisability. 
In Figure 3 it can be seen how the SNR varies for different values of $\alpha$ (real axis). Since the unit disk defines the stability region for the discrete-time case, both $\alpha=-2$ and $\alpha=2$ are unstable cases, but the SNR required for stabilisability is not the same.

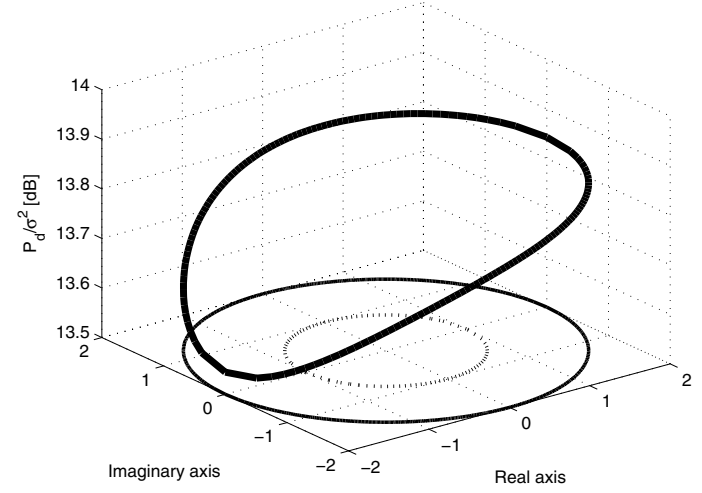

Fig. 3. Signal to Noise Ratio for the case of ACGN channel, thick black line. Unstable plant pole locations, black line, and stability region, dashed black line.

\section{INTERPRETATION OF THE OPTIMAL FEEDBACK SYSTEM}

In this section we assume that the channel is an AWGN channel $(F(z)=1=H(z))$ and that the plant is minimum phase with relative degree one. Notice that in this case (14) will match (3). The resulting connection diagram for the case of the optimal feedback loop using a filtering compensator is shown in Figure 4. After LQG/LTR is performed, the channel input and output satisfy

$$
\begin{aligned}
Y(z) & =S_{o b}(z) C(z I-A)^{-1} W(z)-T_{o b}(z) V(z) \\
Y^{m}(z) & =S_{o b}(z) C(z I-A)^{-1} W(z)+S_{o b}(z) V(z),
\end{aligned}
$$

where $T_{o b}=1-S_{o b}$ and $S_{o b}$ is given in (26).

On the other hand for the optimal predicting observer, see Figure 2, we have that the innovation sequence $e_{k}=$ $y_{k}+v_{k}-C \hat{x}_{k \mid k-1}$ is given by

$$
E(z)=S_{o b}(z) C(z I-A)^{-1} W(z)+S_{o b}(z) V(z),
$$

and that the transfer function relating the estimation error $\tilde{y}_{k \mid k-1}$ with process noise $w_{k}$ and measurement noise $v_{k}$ is

$$
\tilde{Y}(z)=S_{o b}(z) C(z I-A)^{-1} W(z)-T_{o b}(z) V(z) .
$$

Thus, by comparing (31) with (34) and (32) with (34), we can see that the optimal solution for the minimal SNR at the channel input after LTR can be interpreted as a communication channel with feedback from the output to the input. The structure of such feedback is input-output equivalent to an optimal linear quadratic predicting observer. This effectively has the interpretation that the AWGN channel input power minimization performed using Theorem 2 can be seen as an optimal estimation of the AWGN channel output.

\section{CONCLUSION AND REMARKS}

In the present paper we have used LQG/LTR at the output to solve the problem of minimum SNR required for stabilisability of a NMP plant with time delay performed over an ACGN NMP channel model with memory. As a result exact closed loop sensitivity expressions for the optimal solution are obtained for both the case of filtering observer and predicting observer. An interpretation of the optimal SNR stabilizing solution using filtering observer has been given in terms of the predicting estimation of the channel output.

The approach developed here has the potential of being extended to multivariable systems. If the multichannel model of choice is composed by independent ACGN channels with memory and the power constraint is equally shared, then the main difference will be in the dimensions of matrices $B, B_{n}, C$ and $D$ in (14). The validity of such a choice for the multichannel model will be the reason of further investigation.

\section{APPENDIX}

\section{Proof of Theorem 2, part a}

Let $\Phi(z)=(z I-A)^{-1}$. As noted in [15], the filtering compensator can be rewritten as

$$
\begin{aligned}
C_{f}=z K\left(I+\left(I+\Phi K_{f} C A\right)^{-1}\right. & \left.\Phi\left(I-K_{f} C\right) B K\right)^{-1} \\
& \times\left(I+\Phi K_{f} C A\right)^{-1} \Phi K_{f} .
\end{aligned}
$$

Applying Matrix Inversion Lemma and rearranging terms we obtain:

$$
\begin{aligned}
C_{f}=z\left(1+K\left(I+\Phi K_{f} C A\right)^{-1} \Phi\left(I-K_{f} C\right) B\right)^{-1} \\
\times K\left(I+\Phi K_{f} C A\right)^{-1} \Phi K_{f} .
\end{aligned}
$$

Notice that since $C_{a}$ is bicausal the structure of $G(z)$ at infinity is the same when described by $\left(A_{G}, B_{G}, C_{m}\right)$. If recovery is applied using $\left[\begin{array}{lll}C_{G} & 0 & 0\end{array}\right]$ instead of $C$ this will be equivalent to applying recovery using $\left[\begin{array}{lll}C_{m} & 0 & 0\end{array}\right]$ instead of $C$ and, as a result $K$, accordingly with (14), is:

$$
K=\left[\left(C_{m} A_{G}^{l-1} B_{G}\right)^{-1} C_{m} A_{G}^{l} \quad 0 \quad 0\right] .
$$

If we define $H_{m}=\left[\begin{array}{lll}C_{m} & 0 & 0\end{array}\right] A^{l} \Phi K_{f}=C_{m} A_{G}^{l-1} \Phi_{G} K_{p}^{G}$, (in which $K_{p}^{T}$ is factorized as in (22)), it can be seen that (35) becomes

$$
\begin{aligned}
C_{f}=z\left(z C_{m} A_{G}^{l-1} \Phi_{G} B_{G}-z H_{m}(1\right. & \left.+\mathscr{H})^{-1} C \Phi B\right)^{-1} \\
& \times H_{m}(1+\mathscr{H})^{-1} .
\end{aligned}
$$

Again, accordingly to (14), we have $C \Phi B=F C_{G} \Phi_{G} B_{G}=$ $F C_{a} C_{m} \Phi_{G} B_{G}$. Substitute in (37) and rearrange terms:

$$
\begin{aligned}
& C_{f}=\left(C_{m} A_{G}^{l-1} \Phi_{G} B_{G}\right)^{-1} H_{m} \times \\
& \left(1+\mathscr{H}-F C_{a} C_{m} \Phi_{G} B_{G}\left(C_{m} A_{G}^{l-1} \Phi_{G} B_{G}\right)^{-1} H_{m}\right)^{-1} .
\end{aligned}
$$




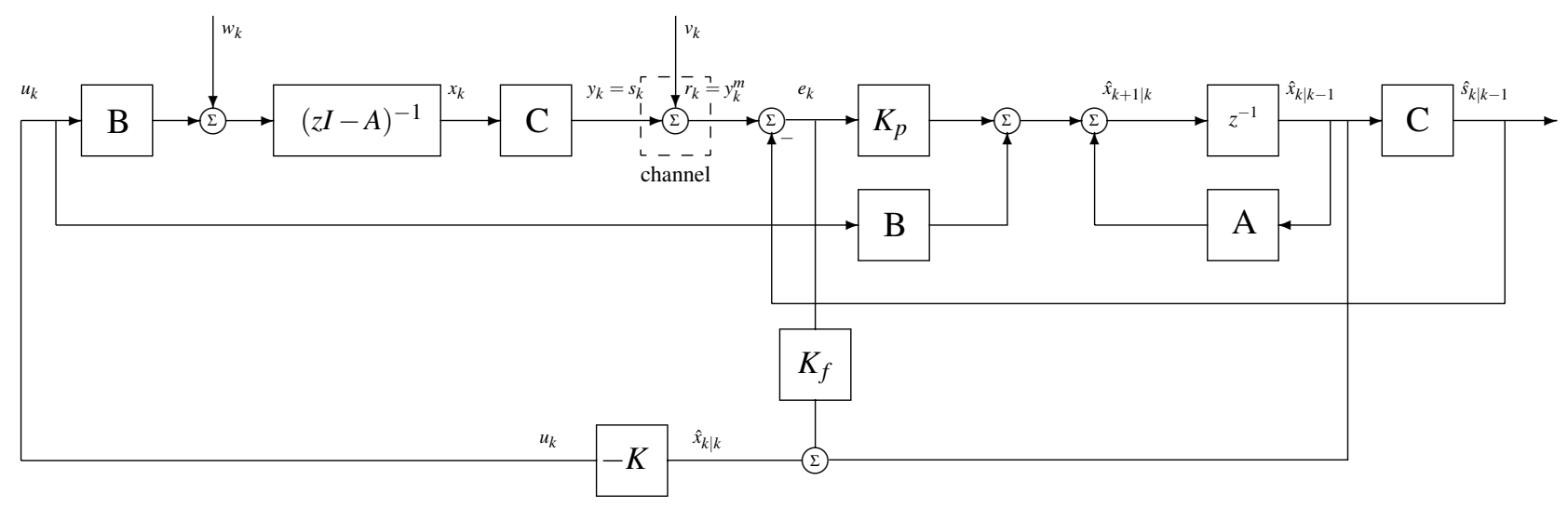

Fig. 4. Feedback system with filtering estimator.

By using the result in (38) and equating $L=F G C_{f}$ (the output open loop transfer function), to $\left(\mathscr{H}-E_{d}\right)\left(1+E_{d}\right)^{-1}$ it is possible to recognize $E_{d}$ as:

$$
E_{d}=\mathscr{H}-F C_{a} C_{m} \Phi_{G} B_{G}\left(C_{m} A_{G}^{l-1} \Phi_{G} B_{G}\right)^{-1} H_{m} .
$$

Replacing definitions for $\mathscr{H}$ and $H_{m}$, using [8, Equation (23)], and factorizing the resulting terms gives (29), which ends the proof.

\section{Proof of Theorem 2, part b}

Let $\Phi(z)=(z I-A)^{-1}$. Consider the definition for the predicting based compensator in (10) and apply matrix inversion lemma:

$$
\begin{aligned}
C_{p}=\left(1+K\left(I+\Phi K_{p} C\right)^{-1}\right. & \Phi B)^{-1} \\
& \times K\left(I+\Phi K_{p} C\right)^{-1} \Phi K_{p} .
\end{aligned}
$$

When loop transfer recovery is applied using $\left[\begin{array}{lll}C_{m} & 0 & 0\end{array}\right]$ instead of $C$, the state feedback gain $K$ will be the same as in (36). Define in this occasion $H_{m}=\left[\begin{array}{lll}C_{m} & 0 & 0\end{array}\right] A^{l} \Phi K_{p}=$ $C_{m} A_{G}^{l} \Phi_{G} K_{p}^{G}$, (in which $K_{p}^{T}$ is factorized as in (22)). Further manipulating (39) gives

$$
\begin{aligned}
C_{p}=\left(z C_{m} A_{G}^{l-1} \Phi_{G} B_{G}-H_{m}(1+\mathscr{H})^{-1}\right. & \left.F C_{a} C_{m} \Phi_{G} B_{G}\right)^{-1} \\
& \times H_{m}(1+\mathscr{H})^{-1},
\end{aligned}
$$

Rearranging terms one last time gives:

$$
\begin{aligned}
& C_{p}=\left(z C_{m} A_{G}^{l-1} \Phi_{G} B_{G}\right)^{-1} H_{m} \times \\
& \left(1+\mathscr{H}-F C_{a} C_{m} \Phi_{G} B_{G}\left(z C_{m} A_{G}^{l-1} \Phi_{G} B_{G}\right)^{-1} H_{m}\right)^{-1} .
\end{aligned}
$$

By using the result in (40) and equating $L=F G C_{f}$ (the output open loop transfer function), to $\left(\mathscr{H}-E_{d}\right)\left(1+E_{d}\right)^{-1}$ it is possible to recognize $E_{d}$ as:

$$
E_{d}=\mathscr{H}-F C_{a} C_{m} \Phi_{G} B_{G}\left(z C_{m} A_{G}^{l-1} \Phi_{G} B_{G}\right)^{-1} H_{m} .
$$

Replacing working definitions for $\mathscr{H}$ and $H_{m}$, using [8, Equation (23)], and factorizing the resulting terms gives (30), which ends the proof.

\section{REFERENCES}

[1] Special Issue on Networked Control Systems. IEEE Transactions on Automatic Control, 49(9), September 2004.

[2] K.J. Åström. Introduction to Stochastic Control Theory. Academic Press, 1970.

[3] A.J. Rojas , J.H. Braslavsky and R.H. Middleton. Output Feedback Control over a Class of Signal to Noise Ratio Constrained Communication Channels. to appear in 2006 American Control Conference, June 2006.

[4] J.S. Freudenberg, J.H. Braslavsky and R.H. Middleton. Control over Signal-to-Noise Ratio Constrained Channels: Stabilization and Performance. In Proceedings 44th IEEE Conference on Decision and Control and European Control Conference, December 2005.

[5] R.H. Middleton, J.H. Braslavsky and J.S. Freudenberg. Stabilization of Non-Minimum Phase Plants over Signal-to-Noise Ratio Constrained Channels. In Proceedings 5th Asian Control Conference, Melbourne, Australia, July 2004.

[6] A. Saberi, B.M. Chen and P. Sannuti. Loop transfer recovery: analysis and design. Springer-Verlag, 1993.

[7] T.M. Cover and J.A. Thomas. Elements of Information Theory. John Wiley \& Sons, 1991.

[8] M. Kinnaert and Y. Peng. Discrete-time LQG/LTR techniques for systems with time delays. Systems and Control Letters, 15:303-311, 1990.

[9] J.M. Maciejowski. Asymptotic Recovery for Discrete-Time Systems. IEEE Transactions on Automatic Control, 30(6):602-605, 1985.

[10] J.H. Braslavsky, R.H. Middleton and J.S. Freudenberg. Feedback stabilisation over signal-to-noise ratio constrained channels. In Proceedings 2004 American Control Conference, Boston, USA, July 2004.

[11] J.H. Braslavsky, R.H. Middleton and J.S. Freudenberg. Effects of Time Delay on Feedback Stabilization over Signal-to-Noise Ratio Constrained Channels. In Proceedings 16th IFAC World Congress, July 2005.

[12] J.H. Braslavsky, R.H. Middleton and J.S. Freudenberg. Feedback Stabilisation over Signal-to-Noise Ratio Constrained Channels. Submitted to IEEE Transactions on Automatic Control, 2005.

[13] G.N. Nair and R.J. Evans. Stabilizability of stochastic linear systems with finite feedback data rates. SIAM J. Control and Optimization, 43(2):413-436, July 2004.

[14] G. Stein and M. Athans. The LQG/LTR procedure for multivariable feedback control design. IEEE Transactions on Automatic Control, 32:105-114, 1987.

[15] Z. Zhang and J.S. Freudenberg. Discrete-time Loop Transfer Recovery for Systems with Nonminimum Phase Zeros and Time Delays. Automatica, 29(2), March 1993.

[16] K. Zhou, J.C. Doyle, and K. Glover. Robust and optimal control. Prentice Hall, 1996. 